\title{
A GENERALIZATION OF NIL-CLEAN RINGS
}

\author{
ALEKSANDRA KOSTIĆ, ZORAN Z. PETROVIĆ, ZORAN S. PUCANOVIĆ, \\ AND MAJA ROSLAVCEV
}

Received 06 April, 2018

\begin{abstract}
The conditions that allow an element of an associative, unital, not necessarily commutative ring $R$, to be represented as a sum of (commuting) idempotents and one nilpotent element are analyzed. Some applications to group rings are also presented.
\end{abstract}

2010 Mathematics Subject Classification: 16U99; 16S34

Keywords: idempotents, nilpotent element, group ring

\section{INTRODUCTION}

An element $a$ in an associative unital ring $R$ is called clean if it can be represented as a sum $a=e+u$, where $e$ is an idempotent element and $u$ is a unit. This notion was introduced by Nicholson in [8]. If one can find such elements $e$ and $u$ such that $a=e+u$ and $e u=u e$, the element $a$ is called strongly clean. The ring $R$ itself is called (strongly) clean if every element in $R$ is (strongly) clean.

Many families of clean rings were investigated in previous decades. In recent years, a particular attention has been paid to the nil-clean rings and its relatives. A nil-clean ring (see [5]) is a ring in which every element is nil-clean, which means that every element can be written as a sum of an idempotent element and a nilpotent one. Analogously, we have a notion of strongly nil-clean elements (and rings). For some of the results concerning this class of rings and some of the related classes of rings, the reader may wish to consult also [1,3,7,10].

A class of strongly 2-nil-clean rings was introduced in [4]. Namely, an element $a$ in a ring $R$ is called strongly 2-nil-clean if it can be represented in the form $a=$ $e+f+n$, where $e$ and $f$ are idempotents, $n$ is a nilpotent element and they all commute with each other.

In this paper we analyze elements of a ring which can be written as a sum of finitely many idempotents and one nilpotent element which are pairwise commutative. If the number of idempotents which appear in this sum is $s$, we call these elements strongly

First, second and fourth author are partially supported by Ministry of Education, Science and Environmental Protection of Republic of Serbia Project \#174032. 
$s$-nil-clean. It turns out that if every element in a ring is strongly $s$-nil-clean for some $s$, this ring has finite characteristic and every element in this ring is strongly $(p-1)$-nil-clean, where $p$ is the largest prime dividing the characteristic of this ring (see Theorem 1$)$. These rings are naturally called strongly $(p-1)$-nil-clean rings and they are all strongly clean (see Corollary 3 and the discussion preceding it). There are many examples of strongly $(p-1)$-nil-clean rings. Theorem 2 shows that in every commutative ring $R$ of finite characteristic $k$, elements which are strongly $s$ nil-clean for some $s$, form a subring which is $(p-1)$-nil clean (where $p$ is the largest prime dividing $k$ ) and if this ring contains idempotents or nilpotents not belonging to $\mathbb{Z}_{k}$ (which is necessarily contained in $R$ ) we have a non-trivial example of such a ring. Proposition 5 provides examples of finite commutative local rings which are $(p-1)$-nil-clean.

The plan of the paper is as follows. In Section 2, we analyze sums of idempotents and one nilpotent element and derive our main criteria for strongly $(p-1)$-nil-clean elements in a ring. Section 3 deals with some structure theorems. It is shown that in analyzing strongly $(p-1)$-nil-clean rings, we may reduce this analysis to the case when $p$ is a nilpotent element in a ring under investigation. We also show in this section that strongly $(p-1)$-nil-clean rings are strongly $\pi$-regular and, consequently, strongly clean.

Section 4 deals with the investigation of group rings $R G$ where $R$ is a $(p-1)$-nilclean commutative ring and $G$ is a group. For example, we show that, for the ring $R G$ to be strongly ( $p-1$ )-nil-clean, when the characteristic of $R$ is of the form $p^{s}$, for a prime integer $p$, it is necessary that the order of any element of a group $G$ is of the form $d p^{k}$, for some $d \mid(p-1)$ and $k \geq 0$ (see Lemma 3). In the case of a commutative group $G$ this condition is also sufficient (see Theorem 4).

We emphasize that we work in associative, unital rings which need not be commutative. When a ring is commutative, we drop the adjective "strongly" since it is unnecessary. We use the same symbol $k$ to denote the integer $k$ and to denote the ring element $k 1_{R}$. It will always be clear what we mean. Finally, we denote the field with $p$ elements by $\mathbb{Z}_{p}$, the Jacobson radical by $J(R)$ and the set of nilpotent elements by $N(R)$.

\section{BASIC RESULTS}

A ring in which every element is a sum of certain number of idempotents and one nilpotent element, that commute with each other, is a generalization of strongly nilclean rings and strongly 2-nil-clean rings. In view of this, we introduce the following definition.

Definition 1. An element $a$ of a ring $R$ is $s$-nil-clean if it can be written in the following form:

$$
a=e_{1}+\cdots+e_{s}+n
$$


where elements $e_{1}, \ldots, e_{s}$ are idempotents and $n$ is nilpotent. If an element $a$ can be written in the form (2.1) so that elements in this sum are pairwise commutative, we say that this element is strongly $s$-nil-clean. If every element in R is (strongly) $s$-nil clean, we say that $R$ is a (strongly) $s$-nil-clean ring.

Of course, if $a$ is (strongly) $s$-nil-clean and $s<t, a$ is also $t$-nil-clean - we simply add $t-s$ zeroes to the presentation of $a$ as a (strongly) $s$-nil-clean element.

Remark 1. It is clear that, if $f: R \rightarrow S$ is a ring homomorphism and an element $a \in R$ is (strongly) $s$-nil-clean, then $f(a) \in S$ is (strongly) $s$-nil-clean. Similarly, an element $a=\left(a_{1}, \ldots, a_{l}\right) \in R_{1} \times \cdots \times R_{l}$ is (strongly) $s$-nil-clean iff $a_{i}$ is (strongly) $s$-nil-clean for all $i$. So, homomorphic images and finite direct products of (strongly) $s$-nil-clean rings are itself (strongly) $s$-nil-clean. Also, a subring of a strongly $s$-nilclean ring has the same property, as we shall see later. However, this does not hold for $s$-nil-clean rings. Namely, if we have a ring which is $s$-nil-clean, but it is not strongly $s$-nil-clean, it is enough to take an element $a$ which is not strongly $s$-nil-clean and look at the subring generated by this element. This subring is commutative, so it cannot be $s$-nil-clean - if it were, this element would also be strongly $s$-nil-clean.

We begin our analysis with a useful result concerning sum of several idempotents and one nilpotent element.

Proposition 1. Let $R$ be a ring and suppose that element $a \in R$ is strongly s-nil clean. Then $a(a-1) \cdots(a-s)$ is nilpotent.

Proof. Let $a=e_{1}+e_{2}+\cdots+e_{s}+n$, where $e_{1}, e_{2}, \ldots, e_{s}$ are idempotents and $n$ is nilpotent that commute with each other. Observe that

$$
1=\left(\left(1-e_{1}\right)+e_{1}\right)\left(\left(1-e_{2}\right)+e_{2}\right) \cdots\left(\left(1-e_{s}\right)+e_{s}\right) .
$$

After multiplication, we get a sum of products of the form

$$
e_{i_{1}} \cdots e_{i_{k}}\left(1-e_{j_{1}}\right) \cdots\left(1-e_{j_{s-k}}\right) \text {, }
$$

where $1 \leq k \leq s, i_{1}<\cdots<i_{k}, j_{1}<\cdots<j_{s-k}$ and $\left\{i_{1}, \ldots, i_{k}, j_{1}, \ldots, j_{s-k}\right\}=\{1, \ldots, s\}$. Next, we get that

$$
\begin{aligned}
& (k-a) e_{i_{1}} \cdots e_{i_{k}}\left(1-e_{j_{1}}\right) \cdots\left(1-e_{j_{s-k}}\right) \\
= & \left(\left(1-e_{i_{1}}\right)+\cdots+\left(1-e_{i_{k}}\right)-e_{j_{1}}-\cdots-e_{j_{s-k}}-n\right) e_{i_{1}} \cdots e_{i_{k}}\left(1-e_{j_{1}}\right) \cdots\left(1-e_{j_{s-k}}\right) \\
= & -n e_{i_{1}} \cdots e_{i_{k}}\left(1-e_{j_{1}}\right) \cdots\left(1-e_{j_{s-k}}\right) .
\end{aligned}
$$

This follows from the fact that $(1-e) e=e-e^{2}=0$, for an idempotent $e$. Since $n$ is nilpotent, so is this product. Thus, when 1 is multiplied by $a(a-1) \cdots(a-s)$, we get a sum of nilpotent elements that commute with each other. Therefore, $a(a-$ 1) $\cdots(a-s)$ is nilpotent.

The following corollary is simple, but important. 
Corollary 1. a) If a ring $R$ is such that -1 is strongly s-nil-clean for some $s \geq 1$, then this ring has finite characteristic.

b) If $\operatorname{char}(R)=k$, then -1 is strongly $(p-1)$-nil-clean, where $p$ is the largest prime dividing $k$.

Proof. a) From the previous proposition we conclude that $(-1)(-2) \cdots(-(s+$ $1))=(-1)^{s+1}(s+1)$ ! is nilpotent, so $((s+1) !)^{m}=0$ for some $m \geq 1$ and the characteristic of the ring $R$ is not 0 .

b) It is enough to show that -1 is $(p-1)$-nil-clean in the ring $\mathbb{Z}_{k}$, which is contained in $R$. If $k=p_{1}^{\alpha_{1}} \cdots p_{l}^{\alpha_{l}}$ is the prime factorization of $k$, where $p_{1}<\cdots<p_{l}=p$,

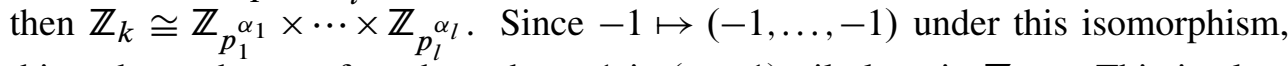
this reduces the proof to show that -1 is $(p-1)$-nil-clean in $\mathbb{Z}_{p_{i}}^{\alpha_{i}}$. This is clear since $-1=p_{i}^{\alpha_{i}}-1=\underbrace{1+\cdots+1}_{p_{i}-1}+p_{i}\left(p_{i}^{\alpha_{i}-1}-1\right)$, and $p_{i}\left(p_{i}^{\alpha_{i}-1}-1\right)$ is nilpotent in $\mathbb{Z}_{p_{i}^{\alpha_{i}}}$

Example 1. The ring $\mathbb{Z}_{k}$ is (strongly) $(p-1)$-nil-clean, where $p$ is the largest prime integer dividing $k$. Namely, using the notation from the previous corollary, this reduces to show that $\mathbb{Z}_{p_{i} \alpha_{i}}$ is $(p-1)$-nil-clean. Since any element in $\mathbb{Z}_{p_{i}}^{\alpha_{i}}$ can be written in the form $\underbrace{1+\cdots+1}_{s}+p_{i} t$, for some $s \in\left\{0, \ldots, p_{i}-1\right\}$ and $t \in$ $\left\{0, \ldots, p_{i}^{\alpha_{i}-1}-1\right\}$, and since $p_{i}$ is nilpotent in $\mathbb{Z}_{p_{i}^{\alpha_{i}}}$, we are done.

Lemma 1. If $k=\operatorname{char}(R)=p_{1}^{\alpha_{1}} \cdots p_{l}^{\alpha_{l}}$ is the prime factorization of the characteristic of the ring $R$, then $R \cong R_{1} \times \cdots \times R_{l}$, where $R_{i}=R / p_{i}^{\alpha_{i}} R$. In particular, $\operatorname{char}\left(R_{i}\right)=p_{i}^{\alpha_{i}}$.

Proof. This follows easily from the Chinese remainder theorem taking into account the fact that elements $p_{i}^{\alpha_{i}}$ are central.

Since the products of the form $a(a-1) \cdots(a-s)$ are important for our investigation, we introduce the symbol $(a)_{k}:=a(a-1) \cdots(a-(k-1))$ (falling factorial, as is known in combinatorics), where $k$ is a positive integer.

We have the following corollary.

Corollary 2. Let $R$ be a ring. Suppose that element $a \in R$ is strongly s-nil clean and $k(<s)$ is nilpotent. Then $(a)_{k}$ is a nilpotent element.

Proof. Clearly

$$
(a)_{s}=a(a-1) \cdots(a-(s-1))=a^{t_{0}}(a-1)^{t_{1}} \cdots(a-(k-1))^{t_{k-1}}+k q(a),
$$

for some non-negative integers $t_{i}$, such that $\sum_{i=0}^{k-1} t_{i}=s$ and polynomial $q(X) \in$ $\mathbb{Z}[X]$. Taking into account that $k$ is nilpotent, the result follows. 
In order to see what the fact that $(a)_{s}$ is nilpotent implies, we start with a simple lemma.

Lemma 2. Let $p$ be a prime integer and $r, m \geq 1$ arbitrary positive integers. Then the element $x$ in the ring $\mathbb{Z}_{p^{r}}[X] /\left\langle X^{m}(X-1)^{m} \cdots(X-(p-1))^{m}\right\rangle$ is (strongly) $(p-1)$-nil-clean, where $x$ is the class of $X$ in this quotient ring.

Proof. Ideals $\langle X-i\rangle$ and $\langle X-j\rangle$ are coprime in $\mathbb{Z}_{p^{r}}[X]$, for all $0 \leq i<j<$ $p$, since $(X-i)-(X-j)=j-i$ and $j-i$ is invertible in the ring $\mathbb{Z}_{p^{r}}[X]$. So, $\left\langle(X-i)^{m}\right\rangle,\left\langle(X-j)^{m}\right\rangle$ are coprime as well. By applying the Chinese remainder theorem we obtain the isomorphism

$\mathbb{Z}_{p^{r}}[X] /\left\langle X^{m} \cdots(X-(p-1))^{m}\right\rangle \cong \mathbb{Z}_{p^{r}}[X] /\left\langle X^{m}\right\rangle \times \cdots \times \mathbb{Z}_{p^{r}}[X] /\left\langle(X-(p-1))^{m}\right\rangle$, such that $x \mapsto(x, \ldots, x)$. Thus, it is enough to show that $x$ has the desired presentation in all factors and since $x=\underbrace{1+\cdots+1}_{i}+(x-i)$ in the factor $\mathbb{Z}_{p^{r}}[X] /\left\langle(X-i)^{m}\right\rangle$, this is true.

Proposition 2. Let $R$ be a ring. Suppose that the element $p$ is nilpotent, where $p$ is a prime integer, and let $a \in R$ be such that $(a)_{p}$ is nilpotent. Then a is strongly $(p-1)$-nil-clean.

Proof. Consider the homomorphism $f: \mathbb{Z}[X] \rightarrow R$, given by $f(X)=a$. An immediate consequence of the fact that $(a(a-1) \cdots(a-(p-1)))^{m}=0$ and that $p^{r}=0$ in $R$, for some $m, r \geq 1$, is the existence of an induced homomorphism

$$
\bar{f}: \mathbb{Z}_{p^{r}}[X] /\left\langle X^{m}(X-1)^{m} \cdots(X-(p-1))^{m}\right\rangle \rightarrow R \text {, such that } x \mapsto a .
$$

Since $x$ is strongly ( $p-1)$-nil-clean, so is its image $a$.

Proposition 3. Let $R$ be a ring of characteristic $k(>0)$. If $p$ is the largest prime dividing $k$ and $a \in R$ is such that $(a)_{s}$ is nilpotent for some $s \geq 1$, then a is strongly $(p-1)$-nil-clean.

Proof. Under isomorphism $R \cong R_{1} \times \cdots \times R_{l}$, where $R_{i}=R / p_{i}^{\alpha_{i}} R$ and $k=$ $p_{1}^{\alpha_{1}} \cdots p_{l}^{\alpha_{l}}$, implied by Lemma 1 , the element $a$ goes to $\left(a_{1}, \ldots, a_{l}\right)$. Also, $a$ is strongly $(p-1)$-nil-clean iff $a_{i}$ is such for all $i$. However, from the fact that $(a)_{s}$ is nilpotent, it follows that $\left(a_{i}\right)_{s} \in R_{i}$ is nilpotent for all $i$. From this, it easily follows that $\left(a_{i}\right)_{p_{i}}$ is also nilpotent for all $i$. Namely, if $s<p_{i}$, this follows since $\left(a_{i}\right)_{p_{i}}=$ $\left(a_{i}\right)_{s}\left(a_{i}-s\right)_{p_{i}-s}$ and if $s>p_{i}$, it follows from Corollary 2. Since $p_{i}$ is nilpotent in $R_{i}$, Proposition 2 gives that $a_{i} \in R_{i}$ is strongly $\left(p_{i}-1\right)$-nil-clean for all $i$. From the fact that $p_{i} \leq p$ for all $i$, it follows that these elements $a_{i}$ are all $(p-1)$-nil-clean and so is $a$.

Theorem 1. Let $R$ be a ring such that every element $a \in R$ is strongly s-nil-clean for some $s$. Then $R$ has finite characteristic and $R$ is strongly $(p-1)$-nil-clean, where $p$ is the largest prime dividing $\operatorname{char}(R)$. 
Proof. Corollary 1 shows that $R$ has finite characteristic and from Proposition 1 and Proposition 3 it follows that every element is strongly $(p-1)$-nil-clean, where $p$ is the largest prime dividing this characteristic.

Theorem 1 shows that one needs only to investigate strongly $(p-1)$-nil-clean rings, where $p$ is a prime integer. For example, the class of all strongly 3-nil-clean rings is the same as the class of strongly 2-nil-clean rings, and the class of strongly 9-nil-clean-rings is the same as the class of strongly 6-nil-clean rings. Namely, if a ring is, say, strongly 9-nil-clean, then $(a)_{10}$ is nilpotent for all $a \in R$. So, this is true for $a=10$. Consequently, $10 !=(10)_{10}$ is nilpotent, so $\operatorname{char}(R) \mid(10 !)^{m}$, for some $m \geq 1$. We conclude that the largest prime dividing $\operatorname{char}(R)$ is at most 7 (it may be smaller), so our ring is strongly 6-nil-clean.

Proposition 4. A subring of a strongly ( $p-1)$-nil-clean ring is also strongly ( $p-$ 1)-nil-clean.

Proof. Let $S$ be a subring of a strongly ( $p-1)$-nil-clean ring $R$ and let $a \in S$. Since $a \in R, a$ is strongly ( $p-1)$-nil-clean, and according to Proposition 1 element $(a)_{p}$ is nilpotent. $R$ is of finite characteristic, say $k$, which means that $\operatorname{char}(S)=k$, with $p$ being the largest prime dividing $k$. When we apply Proposition 3, we get that $a$ is strongly $(p-1)$-nil-clean in $S$.

Theorem 2. Let $R$ be a commutative ring of finite characteristic $k$ and $p$ the largest prime dividing $k$. Let $S=\{a \in R: a$ is s-nil-clean for some $s\}$. Then $S$ is the largest subring of $R$ which is $(p-1)$-nil-clean.

Proof. Since $k=0$ in $R$, we have: $-1=\underbrace{1+\cdots+1}_{k-1}$, so -1 is $(k-1)$-nil-clean and $-1 \in S$. Also, if $a, b \in S$, then $a=e_{1}+\cdots+e_{s}+n, b=f_{1}+\cdots+f_{t}+n^{\prime}$ and we get $a b=\sum_{i, j} e_{i} f_{j}+N$, where $N$ is nilpotent and all $e_{i} f_{j}$ are idempotents ( $R$ is a commutative ring). So, $a b \in S$. Similarly, $a+b=e_{1}+\cdots+e_{s}+f_{1}+\cdots+f_{t}+$ $n+n^{\prime} \in S$. Finally, since $a-b=a+(-1) b$ we conclude that $a-b \in S$ as well. So, $S$ is a ring in which every element is $s$-nil-clean for some $s$. From Theorem 1, we conclude that $S$ is actually $(p-1)$-nil-clean ring. It is clear that $S$ is the largest such subring.

Remark 2. From the Theorem 2 it is clear that, in order to show that a commutative ring of finite characteristic $k$ is $(p-1)$-nil-clean, it is enough to check only its ring generators over $\mathbb{Z}_{k}$. For example, the ring $\mathbb{Z}_{p^{r}}[X] /\left\langle X^{m}(X-1)^{m} \cdots(X-(p-1))^{m}\right\rangle$, appearing in Lemma 2, is $(p-1)$-nil-clean, since it is generated by $x$ and this element is $(p-1)$-nil-clean.

The following proposition provides us with a lot of examples of commutative ( $p-$ 1)-nil-clean rings. 
Proposition 5. Let $R$ be a finite commutative local ring, $M$ its maximal ideal and $R / M \cong \mathbb{Z}_{p}$. Then $R$ is $(p-1)$-nil-clean.

Proof. We know that every element in $M$ is nilpotent. If $x \in R$, then $x+M=$ $s+M$, for some $s \in\{0, \ldots, p-1\}$. So, $x=\underbrace{1+\cdots+1}_{s}+m$, where $m$ is nilpotent.

Remark 3. In the case of non-commutative rings, the set of all elements which are strongly $s$-nil-clean for some $s$ do not necessarily form a subring. Such examples will be given in Section 3 and Section 4. However, a simple application of Zorn's lemma shows that there exist maximal subrings which are strongly $(p-1)$-nil-clean.

The following proposition gives another characterization of strongly $(p-1)$-nilclean elements.

Proposition 6. Let $R$ be a ring of finite characteristic $k, p$ the largest prime dividing $k$ and $a \in R$. The following conditions are equivalent.

(1) $(a)_{p}$ is nilpotent.

(2) a is strongly $(p-1)$-nil-clean.

(3) $a=b+n$, where $b \in R$ is such that $(b)_{p}=0, n$ is nilpotent and $b n=n b$.

Proof. (1) $\Longrightarrow$ (2). This is contained in Proposition 3.

(2) $\Longrightarrow$ (3). Let $a=e_{1}+\cdots+e_{p-1}+n$ be a $(p-1)$-nil-clean decomposition of $a$. Take $b:=e_{1}+\cdots+e_{p-1}$. The proof of Proposition 1 shows that $(b)_{p}=0$.

(3) $\Longrightarrow$ (1). Assume that $a=b+n$. So, we have

$$
(a)_{p}=(b+n)((b-1)+n) \cdots((b-p+1)+n) .
$$

Therefore, since $n$ and $b$ commute, $(a)_{p}=(b)_{p}+n q(n, b)=n q(n, b)$, for some polynomial $q(X, Y) \in \mathbb{Z}[X, Y]$. Since $n$ is nilpotent, so is $(a)_{p}$.

\section{STRUCTURE THEOREMS}

The purpose of this section is to discuss the structure of (strongly) $(p-1)$-nil-clean rings, for prime number $p$.

The following proposition sums up the discussion from the previous section.

Proposition 7. Suppose that $\operatorname{char}(R)=k=p_{1}^{\alpha_{1}} \cdots p_{l}^{\alpha_{l}}$, where $p_{1}<\cdots<p_{l}=p$. Then $R$ is strongly $(p-1)$-nil-clean if and only if $R_{i}$ is strongly $\left(p_{i}-1\right)$-nil-clean, where $R_{i}=R / p_{i}^{\alpha_{i}} R$ and $1 \leq i \leq l$.

This shows that in investigation of strongly ( $p-1)$-nil-clean rings, for $p$ prime, we can reduce our analysis to the case when $p$ is nilpotent (equivalently, when the characteristic of the ring is a power of a prime).

Let us recall that a ring $R$ is called strongly $\pi$-regular if for every element $a \in R$ there exists $n \geq 1$ and $x \in R$ such that $a^{n}=a^{n+1} x$.

Theorem 3. Every strongly $(p-1)$-nil-clean ring is strongly $\pi$-regular. 
Proof. It is enough to consider the case when $p$ is a nilpotent element. Then $(p-1)$ ! is invertible. Let $a \in R$. Since $(a)_{p}$ is nilpotent, we have that $\left((a)_{p}\right)^{s}=0$ for some $s$. But

$$
0=\left((a)_{p}\right)^{s}=(a(a-1) \cdots(a-(p-1)))^{s}=a^{s}((p-1) !)^{s}+a^{s+1} y,
$$

for some $y \in R$. Since $(p-1)$ ! is invertible, we get that $a^{s}=a^{s+1} x$, for some $x \in R$ and we are done.

It is well known that strongly $\pi$-regular ring is strongly clean (see [2, Proposition 2.6], [9, Theorem 1], [5, Corollary 2.4]). Also, Jacobson radical of a strongly $\pi$ regular ring is nil and commutative strongly $\pi$-regular rings have Krull dimension 0 (see [2]). So, we have the following corollary.

Corollary 3. Every strongly ( $p-1)$-nil-clean ring is strongly clean.

The following proposition is rather useful.

Proposition 8. Let $R$ be a ring, $a \in R$ and let $p$ be a nilpotent element, where $p$ is prime. Then $a^{p}-a$ is nilpotent if and only if $(a)_{p}$ is nilpotent.

Proof. It is well-known that $X^{p}-X=(X)_{p}$ in $\mathbb{Z}_{p}[X]$. So, $a^{p}-a-(a)_{p}=$ $\operatorname{pr}(a)$, for some polynomial $r(X) \in \mathbb{Z}[X]$. From this fact, the proof follows immediately.

For future reference, we formulate the following corollary which directly follows from Proposition 6 and Proposition 8.

Corollary 4. Let $R$ be a ring. If $p$ is nilpotent, then $R$ is a strongly $(p-1)$-nilclean ring if and only if $a^{p}-a$ is nilpotent for every $a \in R$.

Let us proceed with some of the special properties of $(p-1)$-nil-clean rings.

Proposition 9. Let $R$ be a ring and let I be any nil ideal of $R$. Then $R$ is $(p-1)$ nil-clean if and only if $R / I$ is $(p-1)$-nil-clean.

Proof. ( $\Longrightarrow$ ) As observed before, this is trivial since $R / I$ is a homomorphic image of $R$.

$(\Longleftarrow)$ Suppose that $R / I$ is $(p-1)$-nil-clean. Let $x \in R$. Then $x+I$ is $(p-1)$ nil-clean. Thus, $x+I=\left(x_{1}+I\right)+\left(x_{2}+I\right)+\cdots+\left(x_{p-1}+I\right)+(y+I)$, where $x_{i}+I$ are idempotents, $1 \leq i \leq p-1$, and $y+I$ is nilpotent. It is well known that idempotents lift modulo nil ideals (see, e.g. [6, Theorem 21.28]) so there are idempotents $e_{i}$ such that $x_{i}+I=e_{i}+I$. So, $x-e_{1}-e_{2}-\cdots-e_{p-1}-y \in I$, i.e., $x=e_{1}+e_{2}+\cdots+e_{p-1}+y+n$, for some $n \in I$. Element $y+n$ is nilpotent. Indeed, since $y+I$ is nilpotent, $y^{k} \in I$ for some $k \in \mathbb{N}$. Every element different from $y^{k}$ in the sum one gets in the expansion of $(y+n)^{k}$, is in $I$ and we can conclude that $(y+n)^{k} \in I$, so $y+n$ is nilpotent. Therefore $R$ is $(p-1)$-nil-clean.

An analogous result holds for the strongly $(p-1)$-nil-clean rings. 
Proposition 10. Let $R$ be a ring and let $I$ be any nil ideal of $R$. Then $R$ is strongly $(p-1)$-nil-clean if and only if $R / I$ is strongly $(p-1)$-nil-clean.

Proof. ( $\Longrightarrow$ ) Again, this is trivial since $R / I$ is a homomorphic image of $R$. $(\Longleftarrow)$ Let $a \in R$. Since $R / I$ is strongly $(p-1)$-nil-clean, one has $\left((a+I)_{p}\right)^{k}=I$, for some $k \in \mathbb{N}$. Consequently, $\left((a)_{p}\right)^{k} \in I$. As $I$ is nil ideal, $\left((a)_{p}\right)^{k} \in N(R)$. So, $(a)_{p}$ is nilpotent. Since $(p)_{p}$ is also nilpotent, the characteristic $k$ of $R$ is finite. The characteristic $l$ of $R / I$ has the property that $p$ is the largest prime dividing this characteristic, but this also holds for $k$. Namely, $l \in I$ and therefore $l$ is nilpotent in $R$. So we have that $k \mid l^{s}$ for some $s$, and also $l \mid k$. It follows that the sets of primes dividing $k$ and $l$ are the same. Now the result follows from Proposition 6.

The following corollary follows directly from the fact that $J(R)$ is nil for a strongly $(p-1)$-nil-clean ring and Proposition 10.

Corollary 5. A ring $R$ is strongly $(p-1)$-nil-clean if and only if $J(R)$ is nil and $R / J(R)$ is strongly $(p-1)$-nil-clean.

\section{GROUP RINGS}

Let us recall the notion of a group ring. Let $G$ be a group, written multiplicatively, and let $R$ be a commutative ring. The group ring of $G$ over $R$, denoted by $R G$, is a free $R$-module with generating set $G$, i.e.:

$$
R G=\bigoplus_{g \in G} R g .
$$

So, elements of $R G$ are formal finite sums of the form $\sum_{i} r_{i} g_{i}$, with $r_{i} \in R, g_{i} \in G$, while the multiplication is implied by multiplication in $G$. The identity of this ring is $1_{R} e$, where $1_{R}$ is the identity in $R$ and $e$ is the neutral element of $G$. We denote the identity simply by 1 .

Our main interest here is focused on strongly $(p-1)$-nil-clean group rings $R G$. It is obvious that if $R G$ is strongly ( $p-1)$-nil-clean, so is $R$. Since we assume that the coefficient ring $R$ is commutative, we refrain from using adjective "strongly" when referring to $R$, we use it only for $R G$ when appropriate. We begin by discussing rings $R$, such that $\operatorname{char}(R)$ is a power of a prime.

Lemma 3. Let $R$ be a $(p-1)$-nil-clean ring such that $\operatorname{char}(R)=p^{s}$, for a prime $p$ and some $s \geq 1$ and let $G$ be a group. For the list of conditions:

(1) $R G$ is strongly $(p-1)$-nil-clean;

(2) For each $g \in G$, the element $g^{p-1}-1$ is nilpotent;

(3) For each $g \in G$, there exists $k \geq 0$ and $d \mid(p-1)$ such that $\omega(g)=d p^{k}$, the following holds: $(1) \Longrightarrow$ (2) and (2) $\Longleftrightarrow$ (3). Here, $\omega(g)$ denotes the order of $g$ in $G$. 
Proof. (1) $\Longrightarrow$ (2). Let $g \in G$. Since $R G$ is strongly ( $p-1)$-nil-clean, from Corollary 4 it follows that $g^{p}-g$ is nilpotent. Since $g$ is invertible, we get that $g^{p-1}-1$ is nilpotent.

(3) $\Longrightarrow$ (2). Let $g \in G$. Then $\omega(g)=d p^{k}$, where $k \geq 0$ and $d \mid(p-1)$. Let $p-1=$ $d s$. Since $\omega\left(g^{d}\right)=p^{k}$ and $\operatorname{gcd}\left(s, p^{k}\right)=1$, we have that $\omega\left(g^{p-1}\right)=\omega\left(\left(g^{d}\right)^{s}\right)=p^{k}$. Therefore,

$$
\left(g^{p-1}-1\right)^{p^{k}}=\underbrace{\left(g^{p-1}\right)^{p^{k}}-1}_{0}+p \cdot u, \text { for some } u \in R G .
$$

Since $p$ is nilpotent, the element $g^{p-1}-1$ is nilpotent as well.

(2) $\Longrightarrow$ (3). Let $g \in G$. The order of $g$ cannot be infinite - in that case, it would not be possible for $g^{p-1}-1$ to be nilpotent. Namely, the element $g^{(p-1) s}$ in the sum $\left(g^{p-1}-1\right)^{s}=g^{(p-1) s}+\cdots+(-1)^{s}$ cannot be cancelled out.

So, let us suppose that $\omega(g)=t p^{k}$, for some $k \geq 0$ and $t$ such that $p \nmid t$ and $\operatorname{gcd}(t, p-1)=d \neq t$. Also, let $t=d t_{1}, p-1=d z_{1}$ and $h=g^{p-1}$. Since $\omega\left(g^{d}\right)=$ $t_{1} p^{k}$ and $\operatorname{gcd}\left(z_{1}, t_{1} p^{k}\right)=1$, it follows that

$$
\omega(h)=\omega\left(g^{p-1}\right)=\omega\left(\left(g^{d}\right)^{z_{1}}\right)=t_{1} p^{k} .
$$

Since $h-1$ is nilpotent, $h^{p^{k}}-1$ is nilpotent as well. Let $h_{1}=h^{p^{k}}$. Then $\omega\left(h_{1}\right)=$ $t_{1}$. Let us focus on the polynomial $f(X)=(X-1)^{t_{1}}-\left(X^{t_{1}}-1\right)$, which is clearly divisible by $X-1$ :

$$
f(X)=(X-1)\left((X-1)^{t_{1}-1}-\left(X^{t_{1}-1}+\cdots+X+1\right)\right)=(X-1) f_{1} .
$$

This follows from the fact that $t_{1} \neq 1$ (which also implies that $h_{1}-1 \neq 0$ ). We can see that

$$
f(X)=(X-1)\left(-t_{1}+(X-1) q(X)\right),
$$

for some polynomial $q \in \mathbb{Z}[X]$, since $f_{1}(1)=-t_{1}$. We can conclude that

$$
f\left(h_{1}\right)=\left(h_{1}-1\right)^{t_{1}}-\underbrace{\left(h_{1}^{t_{1}}-1\right.}_{0})=\left(h_{1}-1\right)\left(-t_{1}+\left(h_{1}-1\right) q\left(h_{1}\right)\right) .
$$

We know that $h_{1}-1$ is nilpotent. As $p \nmid t_{1}$ and $p$ is nilpotent, element $t_{1}$ is invertible in $R$. So

$$
\left(h_{1}-1\right)^{t_{1}}=u\left(h_{1}-1\right),
$$

for an invertible $u \in R G$. So

$$
\left(h_{1}-1\right)\left(\left(h_{1}-1\right)^{t_{1}-1}-u\right)=0,
$$

and since $\left(h_{1}-1\right)^{t_{1}-1}-u$ is invertible, we have that $h_{1}-1=0$. That is a contradiction.

It is easy to check that the proof of $2 \Longleftrightarrow 3$ is valid, although shorter, even for $p=2$. 
In the previous lemma, $G$ was an arbitrary group. If we add commutativity, we actually get equivalence (1) $\Longleftrightarrow$ (2).

Theorem 4. Let $R$ be a $(p-1)$-nil-clean ring such that char $(R)=p^{s}$, for a prime $p$ and some $s \geq 1$ and let $G$ be an Abelian group. Then $R G$ is $(p-1)$-nil-clean iff $g^{p-1}-1$ is nilpotent for every $g \in G$.

Proof. We only need to prove the "if" part. It follows directly from the Remark following Theorem 2 since elements of the group $G$ form a generating set for $R G$ over $R$.

Example 2. The previous theorem does not hold for non-commutative groups. Let us take $R=\mathbb{Z}_{5}$ and $G=\mathbb{D}_{4}$, the dihedral group of order 8 generated by elements $s$ and $r$ such that $s^{2}=1=r^{4}, s r=r^{3} s$. In this group, $g^{4}=1$ for all $g \in G$, so the condition that $g^{4}-1$ is nilpotent is trivially satisfied. However, direct computation shows that

$$
\begin{aligned}
(s+s r)_{5} & =2 s+2 r+3 s r^{2}+3 r^{3} \\
\left((s+s r)_{5}\right)^{8} & =3+2 r^{2} \\
\left(3+2 r^{2}\right)^{2} & =3+2 r^{2},
\end{aligned}
$$

so $(s+s r)_{5}$ is not nilpotent and the group ring $\mathbb{Z}_{5} \mathbb{D}_{4}$ is not strongly 4-nil-clean.

Let us concentrate now on the general case.

Proposition 11. Let $R$ be $a(p-1)$-nil-clean ring and $\operatorname{char}(R)=p_{1}^{\alpha_{1}} \cdots p_{l}^{\alpha_{l}}$, so that $l>1, p_{1}<\cdots<p_{l}=p$.

(1) If $G$ is an elementary Abelian 2-group, then $R G$ is strongly $(p-1)$-nil-clean.

(2) If $G$ is an elementary Abelian group in which every element has order $q$ and $q \mid \operatorname{gcd}\left(p_{1}-1, \ldots, p_{l}-1\right)$, then $R G$ is strongly $(p-1)$-nil-clean.

Proof. (1) As we know, $R \cong R_{1} \times \cdots \times R_{l}$, where $p_{i}$ is nilpotent in $R_{i}$, and consequently $R G \cong R_{1} G \times \cdots \times R_{l} G$. So, all the rings $R_{i}$ are strongly $(p-1)$-nil-clean, and since $p_{i}$ is nilpotent in $R_{i}, R_{i}$ is actually strongly $\left(p_{i}-1\right)$-nil-clean. We will use Theorem 4. Since $2 \mid\left(p_{i}-1\right)$ for all $i \geq 2$ and $g^{2}=1$ for all $g \in G$, we have that $g^{p_{i}-1}-1=0$ for all $g \in G$. If $p_{1}>2$, the same holds for $p_{1}$. If $p_{1}=2$, then $(g-1)^{2}=g^{2}-2 g+1=2(1-g)$. Since 2 is nilpotent in $R_{1} G, g-1$ is also nilpotent in $R_{1} G$. In this case also, from $G$ being Abelian, we can conclude that $R_{i} G$ is $\left(p_{i}-1\right)$-nil-clean. Therefore, $R G$ is $(p-1)$-nil-clean.

(2) Similarly, from $g^{q}=1$, and $q \mid \operatorname{gcd}\left(p_{1}-1, \ldots, p_{l}-1\right)$, we conclude that $g^{p_{i}-1}-$ $1=0$ for all $g \in G$, and proceed as in (1).

Theorem 5. Let $\operatorname{char}(R)=p_{1}^{\alpha_{1}} \cdots p_{l}^{\alpha_{l}}$, where $l>1, p_{1}<\cdots<p_{l}=p$ and let $G$ be a group. Suppose that $R G$ is strongly $(p-1)$-nil-clean ring.

(1) For all $g \in G$ the following holds: $\omega(g) \mid \operatorname{gcd}\left(p_{2}-1, \ldots, p_{l}-1\right)$ and $\omega(g)=$ $d_{1} p_{1}^{s}$, such that $d_{1} \mid\left(p_{1}-1\right)$ and $s \geq 0$. 
(2) If there exists $h \in G$ such that $\omega(h) \geq p_{1}$, then $p_{1} \mid\left(p_{i}-1\right)$, for $2 \leq i \leq l$.

(3) If for all $g \in G, \omega(g)<p_{1}$, then $\omega(g) \mid \operatorname{gcd}\left(p_{1}-1, \ldots, p_{l}-1\right)$.

(4) If $p_{1}=2$, then every element in $G$ has order $2^{s}$ for some $s \geq 0$.

(5) If $p_{1}=2$ and $p_{i} \equiv 3(\bmod 4)$ for at least one $i \geq 2$, then $G$ is an elementary Abelian 2-group.

Proof. (1) As before, $R G \cong R_{1} G \times \cdots \times R_{l} G$, where $p_{i}$ is nilpotent in $R_{i} G$. Lemma 3 shows that $g^{p_{i}-1}-1$ is nilpotent in $R_{i} G$. It follows that $\omega(g)=d_{i} p_{i}^{k_{i}}$ for some $k_{i} \geq 0$ and $d_{i} \mid\left(p_{i}-1\right)$. So,

$$
d_{1} p_{1}^{k_{1}}=d_{2} p_{2}^{k_{2}}=\cdots=d_{l} p_{l}^{k_{l}} .
$$

Since $d_{1}<p_{1}$, it is clear that $d_{1} p_{1}^{k_{1}}$ cannot be divisible by any prime greater than $p_{1}$. So $k_{i}=0$ for $i \geq 2$. Therefore,

$$
\omega(g)=d_{1} p_{1}^{k_{1}}=d_{2}=\cdots=d_{l},
$$

for all $g \in G$, where $d_{i} \mid\left(p_{i}-1\right)$. Hence, $\omega(g)\left|\left(p_{2}-1\right), \ldots, \omega(g)\right|\left(p_{l}-1\right)$, and we are done.

(2) If $h \in G$ is such that $\omega(h) \geq p_{1}$, we have $\omega(h)=d_{1} p_{1}^{k_{1}}=d_{2}=\cdots=d_{l}$, where $k_{1} \geq 1$. Since $d_{i} \mid\left(p_{i}-1\right)$, it follows that $p_{1} \mid\left(p_{i}-1\right)$.

(3) Under this assumption, we get that $k_{1}=0$ in 4.1 , hence for all $g \in G$

$$
\omega(g)=d_{1}=d_{2}=\cdots=d_{l} .
$$

We conclude that $\omega(g) \mid \operatorname{gcd}\left(p_{1}-1, \ldots, p_{l}-1\right)$.

(4) The fourth assertion follows easily. Namely, in this case $p_{1}=2$, so $\omega(g)=d_{1} 2^{k_{1}}$, where $d_{1} \mid(2-1)$. So, $\omega(g)=2^{k_{1}}$.

(5) It is enough to show that there are no elements of order 4 in $G$. If it were, then for an element $g \in G$, we would have equalities

$$
4=d_{2}=\cdots=d_{l},
$$

where $d_{i} \mid\left(p_{i}-1\right)$, for $2 \leq i \leq l$. This would imply that $4 \mid\left(p_{i}-1\right)$, that is $p_{i} \equiv 1$ $(\bmod 4)$, for $2 \leq i \leq l$, which is a contradiction. Hence, we can conclude that $G$ is an elementary Abelian 2-group.

\section{REFERENCES}

[1] S. Breaz, P. Danchev, and Y. Zhou, "Rings in which every element is either a sum or a difference of a nilpotent and an idempotent," J. Algebra Appl., vol. 15, no. 8, p. 11, 2016, doi: 10.1142/S0219498816501486.

[2] W. Burgess and P. Menal, "On strongly $\pi$-regular rings and homomorphisms into them," Comm. Algebra, vol. 16, no. 8, pp. 1701-1725, 1988, doi: 10.1080/00927879808823655.

[3] H. Chen and M. Sheibani, "On strongly nil clean rings," Comm. Algebra, vol. 45, no. 4, pp. 17191726, 2017, doi: 10.1080/00927872.2016.1222411.

[4] H. Chen and M. Sheibani, "Strongly 2-nil-clean rings," J. Algebra Appl., vol. 16, no. 8, p. 12, 2017, doi: 10.1142/S021949881750178X. 
[5] A. Diesl, “Nil clean rings,” J. Algebra, vol. 383, pp. 197-211, 2013, doi: 10.1016/j.jalgebra.2013.02.020.

[6] T. Y. Lam, A first course in noncommutative rings, 2nd ed., ser. Graduate Texts in Mathematics. New York: Springer-Verlag, 2001, vol. 131.

[7] W. McGovern, S. Raja, and A. Sharp, "Commutative nil clean group rings," J. Algebra Appl., vol. 14, no. 6, p. 5, 2015, doi: 10.1142/S0219498815500942.

[8] W. Nicholson, "Lifting idempotents and exchange rings," Trans. Amer. Math. Soc., vol. 229, pp. 269-278, 1977, doi: 10.1090/S0002-9947-1977-0439876-2.

[9] W. Nicholson, "Strongly Clean Rings and Fitting's Lemma," Comm. Algebra, vol. 27, no. 8, pp. 3583-3592, 1999, doi: 10.1080/00927879908826649.

[10] S. Sahinkaya, G. Tang, and Y. Zhou, "Nil-clean group rings," J. Algebra Appl., vol. 16, no. 5, p. 7, 2017, doi: 10.1142/S0219498817501353.

Authors' addresses

Aleksandra Kostić

University of Belgrade, Faculty of Mathematics, Studentski trg 16, Belgrade, Serbia

E-mail address: alex@matf.bg.ac.rs

\section{Zoran Z. Petrović}

University of Belgrade, Faculty of Mathematics, Studentski trg 16, Belgrade, Serbia

E-mail address: zoranp@matf.bg.ac.rs

Zoran S. Pucanović

University of Belgrade, Faculty of Civil Engineering, Bulevar kralja Aleksandra 73, Belgrade, Serbia

E-mail address: pucanovic@grf.bg.ac.rs

Maja Roslavcev

University of Belgrade, Faculty of Mathematics, Studentski trg 16, Belgrade, Serbia

E-mail address: roslavcev@matf.bg.ac.rs 